\title{
Association between autism spectrum disorder and changes in the central auditory processing in children
}

\author{
Laura Faustino Gonçalves ${ }^{1}$ (D), Karina Mary Paiva ${ }^{1}$ (D), Fernanda Soares Aurélio Patatt ${ }^{2}$ (D), \\ Janaina Viana Stolz ${ }^{3} \mathbb{0}$, Patrícia Haas ${ }^{1 *}$
}

\begin{abstract}
$\sqrt{2}$
SUMMARY

OBJECTIVE: To verify the scientific evidence on the association between Autistic Spectrum Disorder and Central Auditory Processing Disorder in children, aiming to answer the following research question: What is the association between Autistic Spectrum and Alteration of Auditory Processing in Children?

METHODS: Studies were chosen through the combination based on the Medical Subject Heading Terms (MeSH): [(auditory processing) and (children) and (autism) and (neurological disorders)]. The MEDLINE (PubMed), LILACS, and SciELO databases were used. The analyzed papers covered a ten-year period, from 2010 to 2020. We selected descriptive, cross-sectional, cohort, and case studies. We evaluated the quality of the papers, which had a minimum score of six in the modified scale of the literature.

RESULTS: 126 papers were retrieved after the exclusion phase, and 17 of them followed the inclusion criteria. Only two papers answered the guiding question with audiological results.

CONCLUSIONS: Patients diagnosed with autistic spectrum disorder may have disturbance central auditory processing, considering that changes were found both in absolute and interpeak latencies in the brainstem evoked response audiometry, as well as in latency and laterality of the N1c wave amplitude. In addition, there were changes in the assessment behavioral auditory processing. Thus, disturbance central auditory processing is common in children with autistic spectrum disorder.
\end{abstract}

KEYWORDS: Autism spectrum disorder. Hearing. Auditory pathways. Auditory perceptual disorders. Speech perception.

\section{INTRODUCTION}

Autistic spectrum disorder (ASD) is defined as a neurodevelopmental disorder, characterized by communication and social interaction difficulties and repetitive or restricted behaviors and/or interests ${ }^{1}$. The prevalence of ASD has increased significantly over the past 20 years. New statistical figures published by the United States Centers for Disease Control and Prevention (CDC) show a prevalence of one autistic person for every 54 children aged eight years ${ }^{2}$.

Among the alterations observed in children with ASD, language is a very important field of interest. In view of the need for the integrity of the auditory pathway from its peripheral to central portion for proper linguistic development, changes in this pathway result in damage to both language acquisition and its functional use ${ }^{3}$.

The set of skills and tasks necessary for individuals to understand what they heard is called central auditory processing (CAP). It encompasses operations performed by the part of the central nervous system (CNS) responsible for analyzing, discriminating, and decoding the sound captured by the ears, thus transforming acoustic energy into an entity with meaning. Individuals with ASD can present alterations in their auditory abilities from perceptual disturbances and difficulties in the communicative abilities associated with such condition. These can culminate in low performance of acoustic information processing in the

\footnotetext{
'Universidade Federal de Santa Catarina - Florianópolis (SC), Brasil.

¿Universidade de Brasília - Brasília (DF), Brasil.

${ }^{3}$ Universidade Federal de Pelotas - Pelotas (RS), Brasil.

*Corresponding author: patrícia.haas@ufsc.br

Conflicts of interest: the authors declare there is no conflicts of interest. Funding: none.

Received on July 23, 2020. Accepted on August 08, 2020.
} 
central auditory nervous system (CANS), that is, resulting in a disturbance central auditory processing (DCAP).

Structural and functional abnormalities that contribute to impaired auditory processing are described in individuals with $\mathrm{ASD}^{4}$. In addition, hearing complaints in children with ASD may be considered part of a higher-order cognitive disorder 5 . Based on the description of auditory problems associated with ASD, the present research has the main and guiding objective of verifying the scientific evidence on the association between ASD and DCAP in children. This paper aims to answer the following research question: "What is the association between ASD and auditory processing alterations in children?".

\section{METHODS}

\section{Search strategy}

We conducted a systematic review according to the recommendations of the Preferred Reporting Items for Systematic Reviews and Meta-Analyses (PRISMA) ${ }^{6}$ guidelines. Two independent researchers searched for scientific papers in the electronic databases MEDLINE (PubMed), LILACS, SciELO, and BIREME from January 2010 to March 2020. The research was structured and organized in the Target population, Intervention, Comparison, Outcomes and Study (PICOS) format (Table 1). Considering the objective of this research, the acronym CONTROL was not used, as it is not applicable.

The keywords were selected from the Health Sciences Descriptors (DeCS) and Medical Subject Heading Terms (MeSH), given their extensive use by the scientific community for indexing articles in the PubMed database. Several keywords and Boolean operators were used for the searches, including (auditory processing and children and autism and neurological disorders), (auditory processing and children and autism and neurological disorders) and randomized controlled trial [pt] OR controlled clinical trial $[\mathrm{pt}]$ OR randomized controlled trials [mh] OR

Table 1. Description of the components of PICOS.

\begin{tabular}{c|c} 
Acronym & Definition \\
\hline P & Child diagnosed with autism \\
\hline I & Speech-language-hearing therapy \\
\hline C & Autism Spectrum Disorder \\
\hline O & Disturbance central auditory processing \\
\hline S & $\begin{array}{r}\text { Descriptive study } \\
\text { Cross-sectional study } \\
\text { Observational study }\end{array}$ \\
\hline
\end{tabular}

Source: developed by the authors. P: population/patients; I: intervention; C: comparison/control; O: outcome; S: study. random allocation [mh] OR double-blind method [mh] OR singleblind method [mh] OR clinical trial [pt] OR clinical trials [mh] OR ("clinical trial”"[tw]) OR ((singl*[tw] OR doubl*[tw] OR trebl*[tw] OR tripl*[tw]) AND (mask*[tw] OR blind*[tw])) OR ("latin square" $[\mathrm{tw}]$ ) OR placebos [mh] OR placebo* [tw] OR random* [tw] OR research design [mh:noexp] OR follow-up studies [mh] OR prospective studies [mh] OR cross-sectional studies [mh] OR control* [tw] OR prospective* [tw] OR volunteer* [tw]) NOT (animal[mh] NOT human[mh]).

The two independent authors identified papers through an electronic search and then organized and reviewed them for duplication. Subsequently, their titles were independently analyzed. Papers that did not meet any inclusion criteria were excluded. In sequence, the abstracts of the papers selected in the second step were examined. Papers that did not contain characteristics of the question to be answered were excluded.

\section{Selection criteria}

We selected descriptive, cross-sectional, cohort, and case studies. Publications were included without any restrictions on language or location. The search comprised the period from January 2010 to March 2020. Studies published in the format of letters to the editor, guidelines, literature reviews, systematic reviews, meta-analyses and abstracts, in addition to publications prior to the year 2010 were excluded. Table 2 represents the inclusion and exclusion criteria for this paper. The studies included patients aged up to 12 years old.

Table 2. Summary of the inclusion and exclusion criteria.

\begin{tabular}{l|c}
\hline \multirow{2}{*}{ Design } & $\begin{array}{c}\text { Case reports } \\
\text { Case-control studies } \\
\text { Controlled clinical trials } \\
\text { Cohort studies } \\
\text { Screening studies } \\
\text { Observational studies }\end{array}$ \\
\hline Place & No restriction \\
\hline Language & No restriction \\
\hline Exclusion & criteria \\
\hline Design & $\begin{array}{c}\text { Letter to the editor } \\
\text { Guidelines } \\
\text { Literature reviews } \\
\text { Systematic reviews } \\
\text { Meta-analyses }\end{array}$ \\
\hline Studies & $\begin{array}{c}\text { Mnclear, poorly described, } \\
\text { or inadequate }\end{array}$ \\
\hline Form of publication & Abstract alone \\
\hline
\end{tabular}

Source: developed by the authors. 


\section{Data analysis}

Data extraction for the eligibility process was performed using a form prepared by the researchers in the Excel program, in which the extracted data were initially added by one of the researchers and then checked by the other. For data obtained from the eligible studies, these data were also transferred to a spreadsheet in the same program in order to organize the results as described in Figure 1.

Subsequently, we verified the methodological quality of the included papers. The resulting score was marked using a protocol for qualitative scoring of the selected studies that was modified based on the literature with scores categorized as of high (between 13 and 11 points), moderate (between 10 and six points), and low (below six points) quality. The included studies were classified as high quality since they scored $\geq 6$. The protocol for qualitative scoring was proposed by Pithon et al. $^{7}$ and modified by the authors.

\section{RESULTS}

One-hundred twenty-six publications were obtained from the keywords. After the exclusion because of duplication, 125 papers remained, of which 108 were excluded after the titles were read. After the initial evaluations, 17 abstracts were reviewed and three complete papers were analyzed from them, of which two were included for answering the guiding reserach question. These two

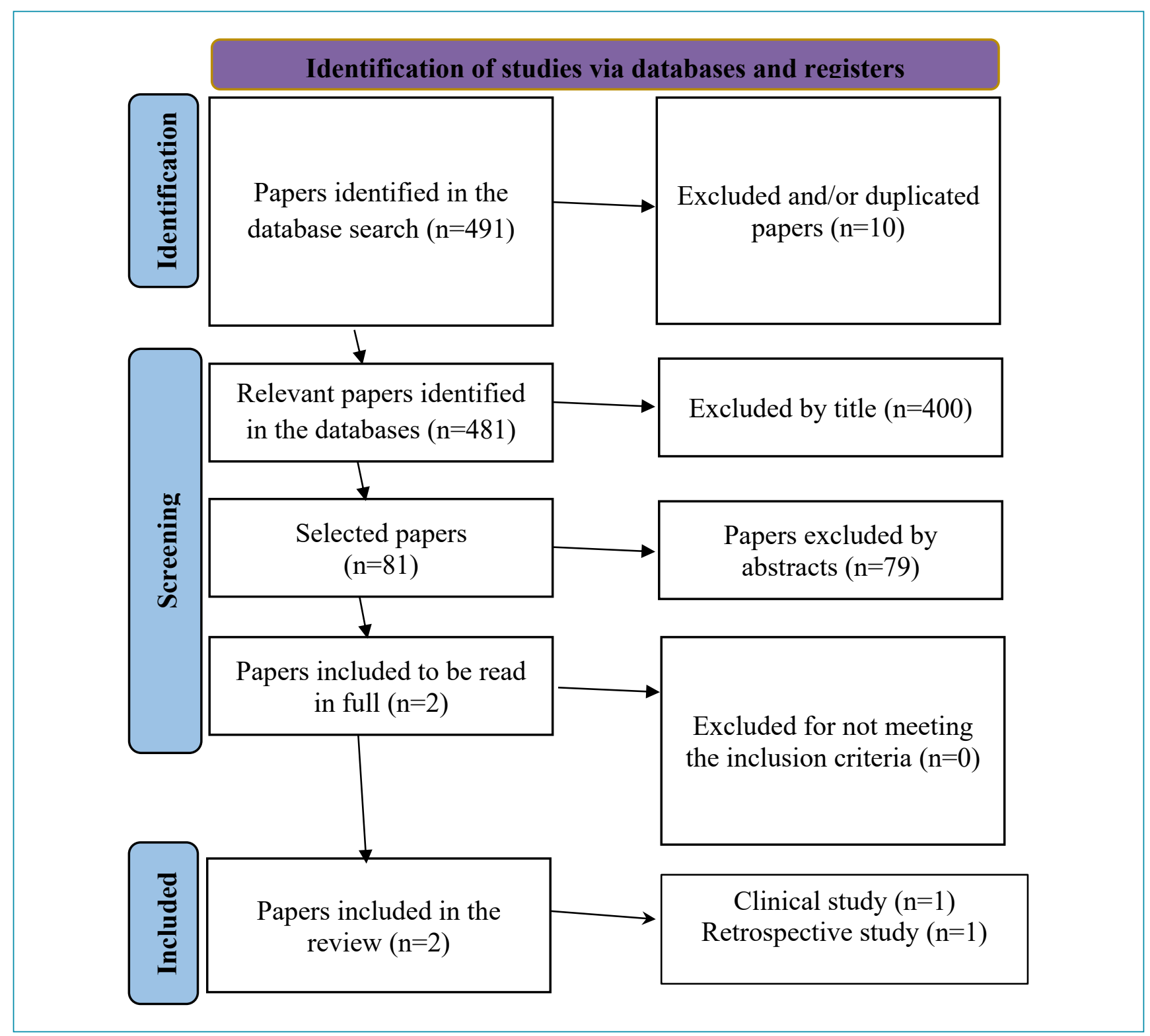

Figure 1. Flowchart of the search, selection, and distribution of the papers. 
papers are descriptive and were developed by researchers from the University of Alexandria, Egypt, which will be referenced according to the chronological order of publication.

The study carried out by Azouz et al. ${ }^{8}$, in which 30 children were diagnosed with ASD, aged between 3 and 7 (76.7\% males and $23.3 \%$ females), was included. It aimed to analyze the auditory profiles at different levels of the auditory system and to verify the role of auditory processing disorder as an essential pathology or an associated comorbidity of autistic disorder. Such study also aimed to establish the correlation between the results of the CAP and the language delay in these cases.

Researchers considered the CAP disorder as consisting of two main components: subcortical and cortical. The subcortical component was studied using the brainstem auditory evoked potential (BAEP), while the cortical one was assessed by the N1 wave response. Although the $\mathrm{N} 1$ response is one of the mandatory components of cortical auditory responses, the study of the N1 wave response parameters, especially their latency, provided the authors with a marker for CAP. The researchers found that $40 \%$ of the participants with ASD were hyper-responsive to auditory stimuli according to the sensory checklist for auditory skills. Furthermore, they verified the absolute latency of the $\mathrm{V}$ wave of the right ear and I wave of the left one. Interpeak latencies I-V and III-V of both ears significantly increased in children with ASD. When comparing the latency of the N1c wave of children with ASD with normative data obtained in 15 children aged 2 to 7 , the researchers investigated the absolute latency of the $\mathrm{N} 1 \mathrm{c}$ wave that was significantly prolonged in the autistic group in both ears, in addition to the amplitude of this larger wave on the right side. Based on these findings, the authors concluded that DCAP is an essential pathology of autistic disorder; therefore, these children have an immature SNAC or dysfunction at the brain and cortical trunk levels ${ }^{8}$.

A study developed by Kozou et al. ${ }^{9}$ was carried out with 30 children diagnosed with ASD, aged between 7 and 12 (16.6\% females and $83.3 \%$ males). It aimed to evaluate various CAP skills in addition to verifying the auditory training effectiveness in these children. In that study, all participants demonstrated normal peripheral hearing levels with medium pure tone thresholds and word recognition thresholds of $25 \mathrm{~dB}$ or less in addition to normal middle ear function. The authors used the dichotic digit test (DDT) to assess the auditory skills that correspond to the test and showed that 14 children with ASD (46.7\%) obtained normal scores in comparison with the normal data of children with typical development, two $(6.7 \%)$ had poor performance in both ears, eight $(26.7 \%)$ had a greater advantage in the right ear together with deficits in the left ear, and six (20\%) showed deficits in the right ear together with a reversal of the advantage in the ear; these six children were left-handed?
The CAP-related skills in children with ASD ranged from completely normal to substantially defective and generally lower than those of children with typical development. Auditory training has been shown to be effective in improving dichotic deficits and other untrained areas of auditory and language processing skills, in children with ASD'. Table 3 summarizes information regarding each study, author(s), year of publication, location, objective, type of study, sample number, age group, tests used to assess the CAP, results, and conclusion. The total sample, based on the sum of the number of individuals from the two selected studies, was 60 participants. Their ages ranged from 3 to 12 years old, and the majority were male.

\section{DISCUSSION}

The present research aimed to verify the possible relationship between ASD and DCAP in children. In studies that analyzed the portion of the children diagnosed with ASD, we found that these children presented some alterations in the CAP. These findings were validated by the increase in the absolute latencies of waves I and V in at least one of the ears, in addition to an increase in interpeak latencies IV and III-V and prolongation of the absolute latency of the N1c wave in both ears ${ }^{8}$. In addition, the scores for CAP-related skills as assessed by DDT ranged from normal to substantially defective and were generally lower than those obtained in individuals with typical development?

Based on these findings, it is noteworthy the importance of developing papers that seek to verify scientific evidence and elucidate the association between ASD and DCAP, especially in children who need anatomical and functional integrity of the auditory pathway for satisfactory linguistic development. It is believed that DCAP may be related to neuropsychiatric disorders, such as ASD, as the auditory function has clinical and neurophysiological similarities with this disorder ${ }^{10}$. Thus, we investigated the hearing ability and conditions of children diagnosed with ASD, in which the BAEP was one of the procedures used by some researchers ${ }^{10-12}$, and it was also demonstrated in one of the selected studies ${ }^{8}$.

Azouz et al. ${ }^{8}$ found BAEP results from children with ASD that presented considered compatibility with the immaturity of the SNAC or with dysfunction in the brain and cortical trunk levels, corroborating with the results found by Kwon et al. ${ }^{10}$. These authors suggested that all children with interpeak prolonged latencies III-V, especially those with high functioning ASD, should be further evaluated for CAP in order to establish a more appropriate treatment plan. Literature reports on the use of BAEP to estimate the risk of ASD have described that this test is routinely performed on newborns who show 
significantly prolonged latencies and that are later diagnosed with ASD, regardless of their hearing thresholds. This finding suggests that abnormal responses can be detected soon after birth ${ }^{13}$. In contrast, literature reports with contradictory results concerning children that underwent BAEP and were diagnosed with ASD describe prolongation, shortening, and absence of abnormalities in the neurotransmission of auditory information ${ }^{14,15}$.

Table 3. Summary of the included papers.

\begin{tabular}{|c|c|c|c|c|c|c|}
\hline $\begin{array}{l}\text { Authors/ } \\
\text { Year/ Place }\end{array}$ & Objective & $\begin{array}{l}\text { Type of } \\
\text { study }\end{array}$ & $\begin{array}{c}\text { Sample } \\
\text { size/Age } \\
\text { group }\end{array}$ & Exams & Results & Conclusion \\
\hline $\begin{array}{l}\text { Azouz et al. }{ }^{8} \text {, } \\
2014 \text {, Egypt }\end{array}$ & $\begin{array}{l}\text { To study the } \\
\text { auditory profile } \\
\text { at different } \\
\text { levels of the } \\
\text { auditory system } \\
\text { in patients with } \\
\text { ASD; to verify } \\
\text { the role of the } \\
\text { CAP disorder } \\
\text { as an essential } \\
\text { pathology in } \\
\text { autism disorder } \\
\text { or an associated } \\
\text { comorbidity, } \\
\text { and to establish } \\
\text { a correlation } \\
\text { between the } \\
\text { findings in the } \\
\text { CAP and the } \\
\text { language delays } \\
\text { in these cases. }\end{array}$ & Descriptive & $\begin{array}{l}n=30 / 7 \text { to } \\
12 \text { years old }\end{array}$ & BAEP & $\begin{array}{l}\text { - } 40 \% \text { of the } \\
\text { participants with ASD } \\
\text { were hyperresponsive } \\
\text { to the auditory stimuli, } \\
\text { according to the auditory } \\
\text { skills' sensory verification } \\
\text { list. } \\
\text { - Absolute latencies and } \\
\text { prolonged interpeak } \\
\text { intervals: V (RE); I (LE); I-V } \\
\text { and III-V (both ears). } \\
\text { - Absolute latency of the } \\
\text { N1c wave significantly } \\
\text { prolonged in the group } \\
\text { with autism, in both ears. } \\
\text { - Greater N1c wave } \\
\text { amplitude on the right } \\
\text { side in individuals with } \\
\text { autism and on the left } \\
\text { side in children with } \\
\text { typical development. }\end{array}$ & $\begin{array}{l}\text { The CAP disorder } \\
\text { is an essential } \\
\text { pathology in } \\
\text { autism disorder. } \\
\text { Patients with } \\
\text { autism either have } \\
\text { a dysfunctional } \\
\text { or immature } \\
\text { CANS or present } \\
\text { a dysfunction at } \\
\text { the cortical and } \\
\text { brainstem levels. }\end{array}$ \\
\hline $\begin{array}{l}\text { Kozou et al. }{ }^{9}, \\
2018, \text { Egypt }\end{array}$ & $\begin{array}{l}\text { This study was } \\
\text { conducted to } \\
\text { assess various } \\
\text { CAP skills in } \\
\text { individuals } \\
\text { with ASD and } \\
\text { to assess the } \\
\text { effectiveness } \\
\text { of auditory } \\
\text { training in } \\
\text { them. }\end{array}$ & Descriptive & $\begin{array}{c}n=30 / 3 \text { to } 7 \\
\text { years old }\end{array}$ & DDT & $\begin{array}{l}\text { - } 46.7 \% \text { of individuals } \\
\text { with ASD achieved } \\
\text { normal scores. } \\
-6.7 \% \text { had a poor } \\
\text { performance in both ears. } \\
\text { - } 26.7 \% \text { had a greater } \\
\text { advantage in the right } \\
\text { ear, along with deficits in } \\
\text { the left ear. } \\
\text { - } 20 \% \text { had deficits in the } \\
\text { right ear, along with a } \\
\text { reverted ear advantage. }\end{array}$ & $\begin{array}{l}\text { The scores } \\
\text { achieved by those } \\
\text { with ASD in the } \\
\text { CAP skills ranged } \\
\text { from normal } \\
\text { to changed } \\
\text { (different degrees } \\
\text { of abnormalities) } \\
\text { and were, in } \\
\text { general, lower } \\
\text { than in individuals } \\
\text { with typical } \\
\text { development. } \\
\text { With the auditory } \\
\text { training, the } \\
\text { participants } \\
\text { diagnosed with } \\
\text { ASD improved their } \\
\text { dichotic deficits, } \\
\text { as well as other } \\
\text { untrained auditory } \\
\text { skill and language } \\
\text { processing areas. }\end{array}$ \\
\hline
\end{tabular}

ASD: autism spectrum disorder; CAP: central auditory processing; n: number in the sample; BAEP: brainstem auditory evoked potentials; RE: right ear; LE: left ear; V: BAEP V wave; I: BAEP I wave; I-V: BAEP I-V waves interpeak interval; III-V: BAEP III-V waves interpeak interval; DDT: dichotic digits test. 
Sixteen individuals with ASD (research group) and 25 with typical development (Control Group), aged between 8 and 20 years, underwent a basic audiological evaluation in addition to BAEP, which found middle latency evoked potential (PEAML), and cognitive auditory evoked potential (P300). All participants had normal hearing thresholds, and ASD individuals showed changes in BAEP and P300, suggesting impairment of the auditory pathway in the brainstem and subcortical and cortical areas ${ }^{12}$. Several other studies that investigated CAP in individuals with ASD also used concurrent electrophysiological measures, consisting mostly of mismatch negativity (MMN) and P300 changes in wave morphology $^{16-25}$. Furthermore, a recent literature review found deterioration in speech perception and expression in patients with ASD, which may also be related to CAP disorders ${ }^{26}$.

For individuals with ASD, the ability to accurately process and interpret auditory information is often difficult. Abnormalities are diverse, ranging from the atypical perception of various lowlevel perceptual characteristics (tone and volume) to the processing of more complex auditory information, such as prosody. These individuals are more likely to present auditory processing deficiencies during complex auditory information processing, which are more serious for speech stimuli than for non-speech stimuli ${ }^{4}$. Another result refers to the N1 amplitude that indicates secondary auditory cortex activation, which was shown to be greater on the right side in children with ASD, while in typical ones, it is usually greater on the left side ${ }^{8}$. In children with ASD, the greater the amplitude of the N1c wave on the right side, the higher the language score, demonstrating that the right hemisphere was the dominant one in the auditory stimuli processing by autistic children.

Kozou et al. ${ }^{9}$ found that the CAP-related skills in children with ASD ranged from normal (46.7\%) to substantially altered and were generally lower than those of children with typical development. Results compatible with normality, both in behavioral assessment and in electrophysiological hearing assessment using BAEP, were also found in a national study conducted with nine individuals with ASD $^{27}$. Normal findings based on BAEP had already been reported in the literature in individuals with $\mathrm{ASD}^{14,28,29}$.

In their research, Kozou et al. ${ }^{9}$ evaluated CAP using TDD, which is a behavioral exam. They verified results from a previous literature paper, in which the same test was used to evaluate three children with ASD and found that one participant (lefthanded) presented a deficit in the right ear in relation to the left ear, and the two right-handed participants demonstrated bilateral deficits in $\mathrm{TDD}^{5}$. In a recent study carried out with a 14-year-old teenager who had been diagnosed with ASD, the researchers evaluated CAP by means of behavioral and electrophysiological exams. In it, the behavioral assessment consisted of the compressed speech (CS), random gap detection test (RGDT), staggered spondaic word test (SSW), frequency and duration pattern tests (TPF and TPD, respectively), and the electrophysiological evaluation performed with the P300. The individuals in this study presented alterations in the auditory abilities of closing, figure-ground, and temporal ordering, in addition to an increase in the latency of the $\mathrm{P} 300$ component ${ }^{25}$.

A lack of studies investigating CAP via behavioral examinations was proposed and performed in one of the texts selected for the development of the present paper'. It is believed that the complexity and subjectivity of the CAP behavioral tests justify the lack of studies using these procedures in children diagnosed with ASD.

However, these tests may be performed at least in children with high functioning or mild degrees of ASD. Studies suggest that these methods may provide valuable information regarding the nature and extent of this disorder and altered auditory skills, in order to establish an intervention program and optimize the linguistic development of the affected children. Studies have shown an improvement in hearing skills in children and adolescents with ASD after auditory training ${ }^{5,9,25}$.

\section{CONCLUSIONS}

Based on the evidence found in the literature, an association between ASD and DCAP is believed to exist since changes were found both in absolute and interpeak latencies, according to the BAEP in addition to the latency and laterality of N1c wave amplitude and changes in the PAC behavioral assessment as found through TDD. Thus, DCAP proves to be an essential finding in children with ASD, whether due to the immaturity of the SNAC or a dysfunction at the brain and cortical trunk levels, thus affecting the processing and interpretation of auditory information and, consequently, linguistic development.

There is still a shortage of studies that investigate CAP through behavioral examinations. Such studies would provide valuable information regarding the nature and extent of the disorder and the altered hearing skills, in order to guide an intervention program and optimize development of the affected children. Therefore, further studies concerning the proposed theme are necessary in order to elucidate the relationship between ASD and DCAP, especially in children, which depend on the anatomical and functional integrity of the auditory pathway for a satisfactory linguistic development.

\section{AUTHORS' CONTRIBUTION}

LFG: Data Curation, Methodology. KMP: Conceptualization. FSAP: Data Curation, Resources. JVS: Validation, Visualization. PH: Conceptualization, Supervision. 


\section{REFERENCES}

1. American Psychiatric Association. Manual de diagnóstico e estatístico de transtornos mentais: DSM-5. 5th ed. Porto Alegre: Artmed; 2014.

2. Maenner MJ, Shaw KA, Baio J, Washington A, Patrick M, DiRienzo $M$, et. al. Prevalence of autism spectrum disorder among children aged 8 years - autism and developmental disabilities monitoring network, 11 sites, United States, 2016. MMWR Morb Mortal Wkly Rep. 2020;69(16):503. https://doi.org/10.15585/mmwr.ss6904a1

3. World Health Organization. Folha informativa: transtorno do espectro autista [Internet]. 2017 [cited on Apr 15, 2017]. Available from: https://www.paho.org/bra/index.php?ltemid=1098

4. O'Connor K. Auditory processing in autism spectrum disorder: a review. Neurosci Biobehav Rev. 2012;36(2):836-54. https:// doi.org/10.1016/j.neubiorev.2011.11.008

5. Denman I, Banajee M, Hurley A. Dichotic listening training in children with autism spectrum disorder: a single subject design. Int J Audiol. 2015;54(12):991-6. https://doi.org/10. 3109/14992027.2015.1070308

6. Moher D, Shamseer L, Clarke M, Ghersi D, Liberati A, Petticrew $M$, et al. Preferred reporting items for systematic review and meta-analysis protocols (PRISMA-P) 2015 statement. Syst Rev. 2015;4(1):1. https://doi.org/10.1186/2046-4053-4-1

7. Pithon MM, Sant'Anna LI, Baião FC, Santos RL, Coqueiro RS, Maia LC. Assessment of the effectiveness of mouthwashes in reducing cariogenic biofilm in orthodontic patients: a systematic review. J Dent. 2015;43(3):297-308. https://doi.org/10.1016/j.jdent.2014.12.010

8. Azouz HG, Kozou H, Khalil M, Abdou RM, Sakr M. The correlation between central auditory processing in autistic children and their language processing abilities. Int J Pediatr Otorhinolaryngol. 2014;78(12):2297-300. https://doi.org/10.1016/.j.jporl.2014.10.039

9. Kozou H, Azouz HG, Abdou RM, Shaltout A. Evaluation and remediation of central auditory processing disorders in children with autism spectrum disorders. Int J Pediatr Otorhinolaryngol. 2018;104:36-42. https://doi.org/10.1016/j.ijporl.2017.10.039

10. Kwon S, Kim J, Choe BH, Ko C, Park S. Electrophysiologic assessment of central auditory processing by auditory brainstem responses in children with autism spectrum disorders. J Korean Med Sci. 2007;22(4):656-9. https://doi.org/10.3346/jkms.2007.22.4.656

11. Roth DA, Muchnik C, Shabtai E, Hildesheimer M, Henklin Y. Evidence for atypical auditory brainstem responses in young children with suspected autism spectrum disorders. Dev Med Child Neurol. 2012;54(1):23-9. https://doi.org/10.1111/j.1469-8749.2011.04149.x

12. Matas CG, Gonçalves IC, Magliaro FCL. Avaliação audiológica e eletrofisiológica em crianças com transtornos psiquiátricos. Rev Bras Otorrinolaringol. 2009;75(1):130-8. https://doi. org/10.1590/S0034-72992009000100021

13. Miron O, Ari-Even Roth D, Gabis LV, Henkin Y, Shefer S, Dinstein I, et al. Prolonged auditory brainstem responses in infants with autism. Autism Res. 2016;9(6):689-95. https://doi.org/10.1002/aur.1561

14. Tharpe AM, Bess FH, Sladen DP, Schissel H, Couch S, Schery T. Auditory characteristics of children with autism. Ear Hear. 2006;27(4):43041. https://doi.org/10.1097/01.aud.0000224981.60575.d8

15. Hyde KL, Samson F, Evans AC, Mottron L. Neuroanatomical differences in brain areas implicated in perceptual and other core features of autism revealed by cortical thickness analysis and voxel-based morphometry. Hum Brain Mapp. 2010;31(4):556-66. https://doi.org/10.1002/hbm.20887
16. Ferri R, Elia M, Agarwal N, Lanuzza B, Musumeci SA, Pennisi $\mathrm{G}$. The mismatch negativity and the P3a components of the auditory event-related potentials in autistic low-functioning subjects. Clin Neurophysiol. 2003;114(9):1671-80. https:// doi.org/10.1016/s1388-2457(03)00153-6

17. Hitoglou M, Ververi A, Antoniadis A, Zafeiriou DI. Childhood autism and auditory system abnormalities. Pediatr Neurol. 2010;42(5):30914. https://doi.org/10.1016/j.pediatrneurol.2009.10.009

18. Haesen B, Boets B, Wagemans J. A review of behavioural and electrophysiological studies on auditory processing and speech perception in autism spectrum disorders. Res Autism Spectr Disord. 2011;5(2):701-14. https://doi.org/10.1016/j.rasd.2010.11.006

19. Ludlow A, Mohr B, Whitmore A, Garagnani M, Pulvermüller F, Gutierrez R. Auditory processing and sensory behaviours in children with autism spectrum disorders as revealed by mismatch negativity. Brain Cogn. 2014;86:55-63. https://doi. org/10.1016/j.bandc.2014.01.016

20. Abdeltawwab MM, Baz H. Automatic pre-attentive auditory responses: $\mathrm{mmn}$ to tone burst frequency changes in autistic school-age children. J Int Adv Otol. 2015;11(1):36-41. https:// doi.org/10.5152/iao.2014.438

21. Donkers FC, Schipul SE, Baranek GT, Cleary KM, Willoughby MT, Evans AM, et al. Attenuated auditory event-related potentials and associations with atypical sensory response patterns in children with autism. J Autism Dev Disord. 2015;45(2):50623. https://doi.org/10.1007/s10803-013-1948-y

22. Vlaskamp C, Oranje B, Madsen GF, Møllegaard Jepsen JR, Durston $S$, Cantio $C$, et al. Auditory processing in autism spectrum disorder: mismatch negativity deficits. Autism Res. 2017;10(11):1857-65. https://doi.org/10.1002/aur.1821

23. Cui T, Wang PP, Liu S, Zhang X. P300 amplitude and latency in autism spectrum disorder: a meta-analysis. Eur Child Adolesc Psychiatry. 2017;26(2):177-90. https://doi.org/10.1007/s00787-016-0880-z

24. Ishikawa M, Itakura S, Tanabe HC. Autistic traits affect P300 response to unexpected events, regardless of mental state inferences. Autism Res Treat. 2017;2017:8195129. https:// doi.org/10.1155/2017/8195129

25. Ferreira L, D'Agostini AR, Pichini FDS, Pazini E, Rechia IC, Biaggio EPV. Auditory training in autism spectrum disorder: a case report. Codas. 2019;31(4):e20180212. https://doi.org/10.1590/23171782/20192018212

26. Ocak E, Eshraghi RS, Danesh A, Mittal R, Eshraghi AA. Central auditory processing disorders in individuals with autism spectrum disorders. Balkan Med J. 2018;35(5):367-72. https:// doi.org/10.4274/balkanmedj.2018.0853

27. Romero ACL, Gução ACB, Delecrode CR, Cardoso ACV, Misquiatti ARN, Frizzo ACF. Avaliação audiológica comportamental e eletrofisiológica no transtorno do espectro do autismo. Rev CEFAC. 2014;16(3):707-14. https://doi.org/10.1590/1982-021620140313

28. Coutinho MB, Rocha V, Santos MC. Auditory brainstem response in two children with autism. Int J Pediatr Otorhinolaryngol. 2002;66(1):81-5. https://doi.org/10.1016/s0165-5876(02)00211-2

29. Russo N, Nicol T, Trommer B, Zecker S, Kraus N. Brainstem transcription of speech is disrupted in children with autism spectrum disorders. Dev Sci. 2009;12(4):557-67. https://doi. org/10.1111/j.1467-7687.2008.00790.x 